\title{
MINIMIZAÇÃO DE VIBRAÇÕES TORCIONAIS EM COLUNAS DE PERFURAÇÃO DE POÇOS DE PETRÓLEO POR LEIS DE CONTROLE EM FUNÇÃO DO PESO NA BROCA.
}

\author{
HUGO L. S. MONTEIRO ${ }^{1}$, MARCELO A. TRINDADE ${ }^{1}$.
}

\author{
1. Laboratório de Dinâmica, Escola de Engenharia de São Carlos, Universidade de São Paulo \\ Secretaria de Pós - Graduação do Programa de Engenharia Mecânica da EESC/USP \\ Av. Trabalhador SãoCarlense, 400, CEP: 13566-590, São Carlos - SP \\ E-mails: hugo.monteiro@yahoo.com.br; trindadeesc.usp.br.
}

\begin{abstract}
The stick-slip phenomenon, in the process of drilling oil wells, due to the interaction between drill and rock formation can lead to large fluctuations in drill-bit angular velocity and, thus, cause irreparable damage to the process. In this work, the performance of control laws applied to the rotary table (responsible for moving the drill string) is analyzed, in order to reduce stick-slip and drill-bit angular velocity oscillations. The control laws implemented are based on a PI (ProportionalIntegral) controller, for which the torque applied to the rotating table has components proportional and integral to table angular velocity with constant or variable WOB (Weight On Bit). For the drillstring, a finite element model with a linear interpolation was proposed. The torque on the drill-bit was modeled by a non-regularized Coulomb friction model, with parameters that were adjusted using empirical data proposed in literature. Several performance criteria were analyzed and it was observed that the minimization of the mean deviation of the drill-bit angular velocity relative to the target one would provide the best operating condition. Parametric analyses of proportional and integral control gains were performed, yielding level curves for the mean deviation of drill-bit angular velocity. From these curves, stability regions were defined in which the deviation is acceptable. These regions were observed to be wider for smaller values of WOB and higher values of target angular velocity and vice-versa. The inclusion of a controlled dynamic WOB reduced the levels of mean deviation of angular velocity, leading to improved stability regions for the drilling process.
\end{abstract}

Keywords: Oil well drilling, torsional vibration control, stick-slip, drill-string dynamics.

\begin{abstract}
Resumo - O fenômeno de stick-slip, no processo de perfuração de poços de petróleo, é propiciado pela interação entre broca e formação rochosa e pode dar origem a grandes oscilações na velocidade angular podendo provocar danos irreparáveis ao processo. Neste trabalho, analisa-se o desempenho de leis de controle aplicadas à mesa rotativa (responsável por movimentar a coluna de perfuração), visando à redução de stick-slip e de oscilações da velocidade angular da broca. As leis de controle implementadas são do tipo PI (Proporcional-Integral), com parcelas de torque aplicado à mesa rotativa, proporcional e integral à velocidade da mesa, podendo ser com peso na broca constante ou variável. Para a coluna de perfuração, foi proposto um modelo em elementos finitos com função de forma linear. O torque na broca foi modelado segundo atrito de Coulomb pela forma não regularizada, curva esta ajustada pelos dados empíricos conforme propostas da literatura. Diversos critérios de desempenho foram analisados e foi observado que a minimização do desvio médio da velocidade angular em relação à referência propicia melhores condições de operação. Análises paramétricas dos ganhos de controle proporcional e integral foram realizadas, dando origem a curvas de nível para o desvio médio de velocidade angular na broca. A partir destas curvas, foram definidas regiões de estabilidade nas quais o desvio é aceitável. Estas regiões foram observadas serem maiores para menores pesos na broca e maiores velocidades angulares de referência e vice-versa. A adição do controle do peso na broca permitiu uma redução global dos níveis de desvio médio de velocidade angular, dando origem a um aumento das regiões de estabilidade do processo de perfuração.
\end{abstract}

Palavras-chave - Perfuração de poços de petróleo, controle de vibrações torcionais, fenômeno stick-slip, dinâmica de colunas de perfuração.

\section{Introdução}

O processo de perfuração de poços de petróleo consiste, de acordo com Jansen e van den Steen (1995), na abertura de poços em formações rochosas, com diâmetros de cerca de 10 a $85 \mathrm{~cm}$ e profundidades de 0-5000 metros para a extração de petróleo e gás.

A perfuração rotativa ocorre, de acordo com Mihajlovic et al (2006), pela rotação da broca (drill bit), que cria o caminho para um poço (borehole). Esta broca é componente de um segmento pesado denominado BHA (Bottom Hole Assembly), cuja ligação com a superfície é feita por uma coluna de perfuração (drillstring), que consiste, segundo
Tucker e Wang (1999a) de uma série de tubos cilíndricos de aço conectados. A coluna de perfuração e o BHA formam, basicamente, o conjunto de perfuração, que é rotacionado pelo torque motor fornecido pela mesa rotativa (rotary table), na superfície.

Durante o processo de perfuração, vibrações axiais, torcionais e laterais, ou combinações destes, são geradas levando a um dos principais problemas no processo de perfuração de poços de petróleo (Schlumberger, 2006).

Evidências experimentais manifestam que a vibração em colunas de perfuração é uma das maiores causas de deterioração no processo de perfuração; se não controlada, pode resultar em: perda de eficiência, falha prematura dos 
componentes, mudanças inesperadas na direção da perfuração, e até mesmo quebrar a coluna, inutilizando aquele poço. (KHULIEF e ALNASER, 2005).

As vibrações torcionais podem causar variação da rotação ao longo da coluna, danificar as brocas e prejudicar o processo de perfuração, segundo Álamo (2004), e podem gerar, segundo Plácido, Santos e Galeano (2002), o fenômeno de stick-slip, que é uma das principais causas de falhas no processo de perfuração.

O stick-slip é caracterizado pela parada da broca (parte inferior), e do conjunto BHA, enquanto a mesa giratória (em cima) continua girando e transmitindo potência, de modo que, aumenta-se a energia de deformação de torção armazenada na coluna de perfuração e, portanto, o torque aplicado à broca, até que a broca supera a resistência de atrito entre a broca e a formação rochosa e começa a perfurar novamente, mas podendo atingir velocidades muito mais altas do que a esperada.

Em medições de campo, apresentadas por Plácido, Santos e Galeano (2002), o stick-slip é observado por fortes oscilações na velocidade de perfuração. Essa oscilação é auto-excitada se tornando regular e estável uma vez que o modo é iniciado e é reduzida se a velocidade é aumentada (Plácido, Santos e Galeano, 2002; Trindade e Sampaio, 2005). A frequência destas oscilações é geralmente um pouco abaixo da primeira freqüência natural de vibração de torção na faixa de 0,05 a 0,5 Hz (Jansen e Van den Steen, 1995).

Apesar de a propagação das ondas de torção ser regida por equações de onda lineares, a interação entre broca e formação rochosa apresenta um comportamento altamente não-linear que pode ser uma causa de regime não-uniforme de penetração (Spanos et al., 1995, Tucker e Wang, 2003). Richard e Detournay (2000) estudaram a resposta autoexcitada de tais sistemas usando um modelo discreto com dois graus de liberdade (DOF) para demonstrar que o acoplamento linear entre os modos normal e tangencial na broca são suficientes para gerar o fenômeno de stick-slip.

Ritto, Soize e Sampaio (2009) também estudaram a interação não linear entre broca e formação rochosa, usando, porém, um modelo estocástico para as principais forças que atuam sobre o sistema.

Jansen e Van den Steen (1993) adotaram um modelo simplificado de atrito que captura a essência do efeito nãolinear e parece ser suficiente para realizar análises lineares. Tucker e Wang (1999b) utilizaram uma abordagem contínua para a coluna combinada a um modelo de atrito entre broca e formação rochosa que é uma função do movimento relativo. Uma vez que vibrações induzidas pelo fenômeno de stick-slip têm um papel fundamental no processo de perfuração, várias técnicas diferentes têm sido propostas ao longo dos anos para controlar este fenômeno. Elas podem ser resumidas nas seguintes categorias: amortecimento ativo (Jansen e Van den Steen, 1993), retificação de torque (Tucker e Wang, 1999a), "torque suave" (soft-torque) (Tucker e Wang, 1999a; Tucker e Wang, 2003), controle proporcional-integral (PI) de velocidade (Christoforou e Yigit, 2003; Trindade e Sampaio, 2005) e variação dinâmica do peso na broca (Lopez e Suarez, 2004).

Neste trabalho, consideram-se duas modelagens matemáticas para o fenômeno do atrito, baseadas ambas no atrito de Coulomb, que, como descrito anteriormente, apresenta uma descontinuidade em torno do zero, quando se trata a força de atrito em função da velocidade de contato entre as partes; no caso, a velocidade da broca em relação à formação rochosa.

A proposta de atrito regularizado em zero elimina matematicamente a descontinuidade, tornando nula a força de atrito quando a velocidade de deslocamento entre as partes torna-se zero. E, para velocidades em torno do zero, têm-se, igualmente, baixos valores para o módulo da força.

Para a proposta de atrito não regularizado, no entanto, a descontinuidade é trabalhada matematicamente para a velocidade entre as partes, considerando-se um pequeno intervalo em torno do zero no qual a função de atrito fica bem definida.

Manzatto (2011) utiliza da formulação de atrito não regularizado e analisa o modelo com um controlador proporcional integrativo (PI), e propõe critérios de análise de desempenho do processo de perfuração, que são os mesmos utilizados neste trabalho.

Coletti (2011) estuda o mesmo modelo trabalhando com três técnicas de controle, um controlador PI simples e, adicionalmente, duas implementações: a variação do peso na broca e o soft torque, proposto por Tucker e Wang (2003).

São utilizadas, ainda, as seguintes modelagens propostas: para a coluna de perfuração, de um modelo em elementos finitos, com função de forma linear; para o torque na broca, de um modelo de atrito não regularizado, proposta feita por NavarroLópez e Suárez (2004), utilizada também por Manzatto (2011) e Coletti (2011), e ajustada pelos dados empíricos fornecidos por Tucker e Wang (2003).

Com estas definições, pretende-se, neste trabalho, analisar o desempenho destas leis de controle, na correção e/ou eliminação de vibrações torcionais e do fenômeno stick-slip, presentes no processo de perfuração de poços de petróleo. 


\section{Propósito}

Obteve-se um modelo físico-matemático para o conjunto de perfuração, considerando-se os elementos: mesa rotativa (Rotary Table), que aplica o torque motor ao conjunto de perfuração, sendo que é onde foram aplicadas as leis de controle, à velocidade da mesa; coluna de perfuração (Drill String), elo de ligação entre a mesa e a broca; e a broca (Bit), sendo que a broca, neste caso, representa o conjunto denominado $B H A$ (Bottom Hole Assembly), que recebe o torque resistivo, oriundo da interação broca e formação rochosa.

O conjunto é descrito por equações que acopladas formam uma equação matricial, de acordo com a equação 1 , sendo $\mathbf{u}$ o vetor de deslocamentos angulares nodais e $\mathbf{M}, \mathbf{C}$ e $\mathbf{K}$ as matrizes de massa, amortecimento e rigidez. O vetor $\mathbf{F}$ contém os torques aplicados na mesa $(\mathrm{Tm})$, no primeiro nó e na broca $(T b)$, no último nó. (MONTEIRO e TRINDADE, 2012).

$$
[M]\{\ddot{u}\}+[C]\{\dot{u}\}+[K]\{u\}=\{F\}
$$

\subsection{Modelagem do torque na mesa}

A velocidade da mesa é a variável a ser controlada para este modelo de sistema de perfuração.

O controlador utilizado é do tipo proporcional integrativo, o qual imprime um fator de ganho proporcional $K p$ à diferença entre a velocidade da mesa e a velocidade de referência para o conjunto, e um ganho integrativo, $K i$, que multiplica a diferença entre o deslocamento real (integral da velocidade) e o deslocamento esperado. Este controlador é não colocalizado, pois apenas responde às variações na mesa e não atua diretamente na broca, e sim, indiretamente.

Este controlador é descrito pela equação 2.

$$
T_{m}=K_{p}\left(\omega_{r e f}-\omega_{m}\right)+K_{i}\left(\omega_{r e f} t-\omega_{m}\right)
$$

Sendo que $K p$ e $K i$ são os parâmetros proporcional e integral de controle, respectivamente, e ${ }^{\omega_{\text {ref }}}$ a velocidade de referência adotada, enquanto $\omega_{m}$ é a velocidade da mesa, $\theta_{m}$ o deslocamento angular da mesa (topo da coluna), e $T_{m}$ o torque na mesa, que movimenta a coluna de perfuração.

\subsection{1 $\quad 1^{\text {a }}$. Lei de Controle}

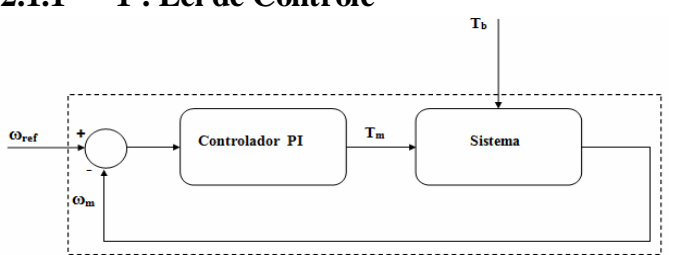

Figura 1 - Modelo esquemático de um controlador PI com peso na broca constante, adotado para o sistema de perfuração.
$\mathrm{Na}$ figura 1, apresenta-se um modelo esquemático para esta $1^{\mathrm{a}}$. Lei de Controle considerada, em que: $T_{b}$, o torque na broca, proveniente da interação da broca com a formação rochosa, e $\omega_{\text {ref }}$, velocidade angular de referência, representam as entradas do sistema, enquanto que $\omega_{m}$, variável de controle, representa a saída. $T_{m}$ é a saída do controlador, torque que deve ser aplicado pela mesa rotativa.

A função de torque $T_{b}$ depende do peso atribuído na broca (WOB, Weight On Bit). Este controlador proporcional integrativo para $W O B$ constante constitui a primeira lei de controle considerada: Controlador Proporcional Integrativo com peso na broca constante.

\subsection{Modelagem do torque na broca}

\subsubsection{Proposta de torque na broca não regularizado}

Foi utilizada a proposta de Navarro-López e Suárez (2004) para a modelagem, segundo atrito de Coulomb, do torque esperado na broca, conforme mostrada na figura 2 .

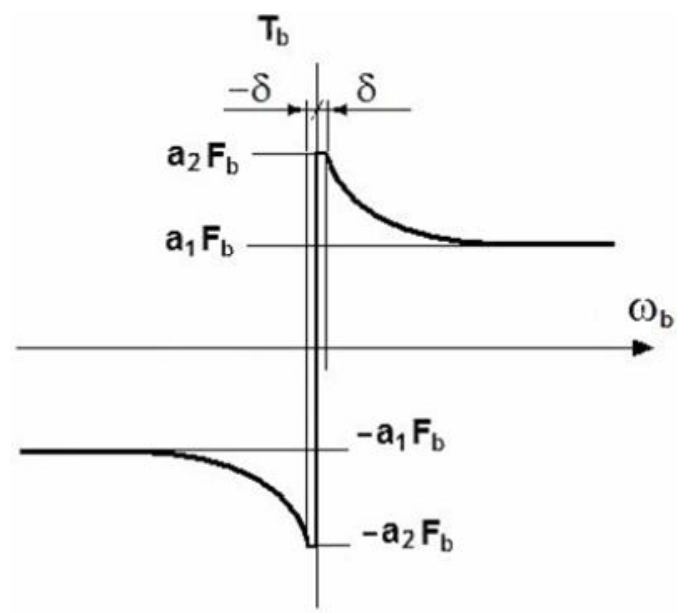

Figura 2 - Modelo de torque na broca segundo atrito de Coulomb, de acordo com proposta de Navarro-López e Suárez (2004), por Manzatto (2011).

Esta função é definida em três intervalos:

$1^{\circ}$. Para velocidades angulares na broca com valor absoluto não nulo, ou maior do que um tolerância de valor $\delta$ (neste trabalho, 0.0001), o torque decai segundo uma função exponencial de limite de atrito estático até um valor de convergência de atrito dinâmico (equação 3).

$2^{\circ}$. Para velocidades menores do que $\delta$, cujo torque não ultrapassou o torque devido ao atrito estático, o valor do torque é a constante de rigidez do elemento finito adjacente à broca multiplicada pela diferença de deslocamento angular entre as extremidades do elemento (equação 4). 
$3^{\circ}$. Se a velocidade na broca é menor do que $\delta$ e o torque na broca é maior do que o limite estático, o torque na broca torna-se igual ao torque de atrito estático, correspondente à multiplicação de um coeficiente $\left(a_{2}\right)$ pelo peso na broca $\left(F_{b}\right)$ (equação 5).

$$
\begin{aligned}
& T_{b}=K\left(\theta_{n-1}-\theta_{n}\right) ;\left|\omega_{b}\right| \leq \delta \text { e }|T| \leq a_{2} F_{b} ; \\
& T_{b}=a_{2} F_{b} \operatorname{sign}(T) ;\left|\omega_{b}\right| \leq \delta \text { e }|T|>a_{2} F_{b} ; \\
& T_{b}=\left[a_{1}+\left(a_{2}-a_{1}\right) e^{-\beta\left|\omega_{b}\right|}\right] F_{b} \operatorname{sign}\left(\omega_{b}\right) ; \text { e }\left|\omega_{b}\right|>\delta
\end{aligned}
$$

Em que: $K$ é a constante de rigidez da coluna em relação à torção, $G J / L ; \theta_{n-1}$ é o deslocamento angular da extremidade adjacente à broca; $\theta_{n}$ é o deslocamento angular da extremidade da broca.; $\operatorname{sign}\left(\omega_{b}\right)$ é a função sinal da velocidade angular na broca; $a_{1}$ é o coeficiente multiplicador menor, em similaridade com o atrito dinâmico. $a_{2}$ é o coeficiente multiplicador maior, em similaridade com o atrito estático; $\beta=$ Expoente de decaimento; e $F_{b}$ é o Peso na broca, $W O B$.

\subsection{Ajuste dos coeficientes $a_{1}, a_{2}$ e $\beta$}

Foram utilizados dos valores típicos dos parâmetros de perfuração apresentados por Tucker e Wang (2003), e uma típica condição de perfuração foi simulada, para a qual a velocidade angular de referência é de $100 \mathrm{rpm}(10.47 \mathrm{rad} / \mathrm{s})$ e, o peso na broca, $120 \mathrm{kN}$. Os valores dos parâmetros de controle proporcional-integrativo são $K p=$ 200 N.m.s e $K i=100$ N.m e a velocidade angular inicial de $70 \mathrm{rad} / \mathrm{s}$ em toda a coluna. Foi considerado o intervalo de tempo para análise de 0 a 100 s.

Ajustou-se, segundo estes dados empíricos, a função de decaimento descrita na equação 4, comparando-a às curvas obtidas para esta função regularizada em zero, encontrada por Trindade e Sampaio (2005), para cada peso na broca, para se obter os coeficientes $a_{1}$ e $a_{2}$, e também o expoente de decaimento $\beta$.

Os valores obtidos para os coeficientes $A, B$, $a_{1}, a_{2}$ e $\beta$ para os pesos na broca considerados (80, $100,120,140$ e $160 \mathrm{kN})$, foram registrados na tabela 2.

Tabela 1 - Coeficientes $A, B, a_{1}, a_{2}, \beta$, para a função modeladora do torque na broca não regularizada, para os valores de pesos na broca: $80,100,120,140$ e $160 \mathrm{kN}$.

\begin{tabular}{cccccc}
\hline \multicolumn{6}{c}{$W O B(\mathrm{kN})$} \\
\hline $\begin{array}{c}\text { Coeficiente } \\
\mathrm{s}\end{array}$ & 80 & 100 & 120 & 140 & 160 \\
\hline$a_{1}$ & 0,037 & 0,032 & 0,029 & 0,026 & 0,025 \\
$a_{2}$ & 0,057 & 0,070 & 0,079 & 0,085 & 0,089 \\
$\beta$ & 0,082 & 0,093 & 0,097 & 0,098 & 0,099 \\
\hline
\end{tabular}

A partir dos valores constantes na tabela 2 , para os coeficientes $a_{1}, a_{2}$, e $\beta$, obtiveram-se os gráficos do comportamento do torque na broca em relação à velocidade angular na broca, para este modelo de torque na broca não regularizado, nas curvas em vermelho, e para a função descrita na equação 31, por Trindade e Sampaio (2005), em preto.

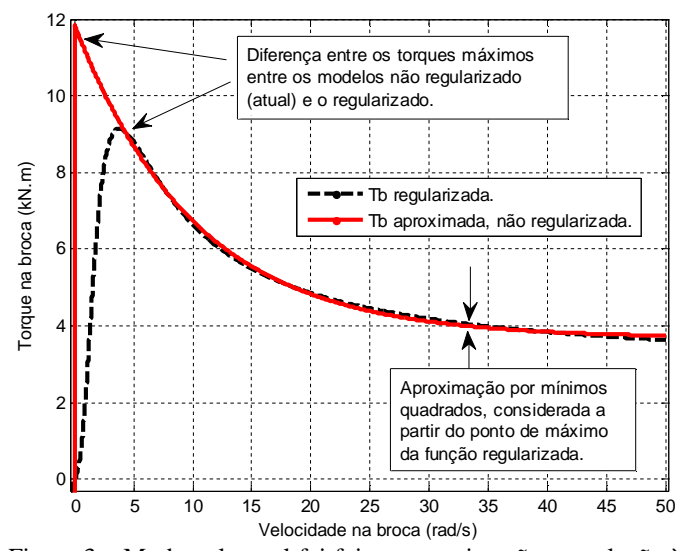

Figura 3 - Modo pelo qual foi feita a aproximação em relação à proposta deste trabalho, em vermelho, pela função de torque regularizada por Trindade e Sampaio (2005), para o intervalo de velocidades na broca de 0 a $50 \mathrm{rad} / \mathrm{s}$.

\subsection{Modelo proposto}

Propõe-se o modelo descrito pela figura 4. Duas inércias, a inércia da mesa rotativa, $J_{m}$, e do conjunto da broca (BHA), $J_{b}$, encontram-se ligadas, por um haste flexível à torção (coluna de perfuração), rígida axialmente, cujo movimento considerado foi apenas a rotação, em torno do próprio eixo axial. Apenas duas forças foram consideradas atuantes no sistema: o torque motor, na mesa, $T_{m}$, e o torque propiciado pelo atrito, de resistência, na broca, $T_{b}$.

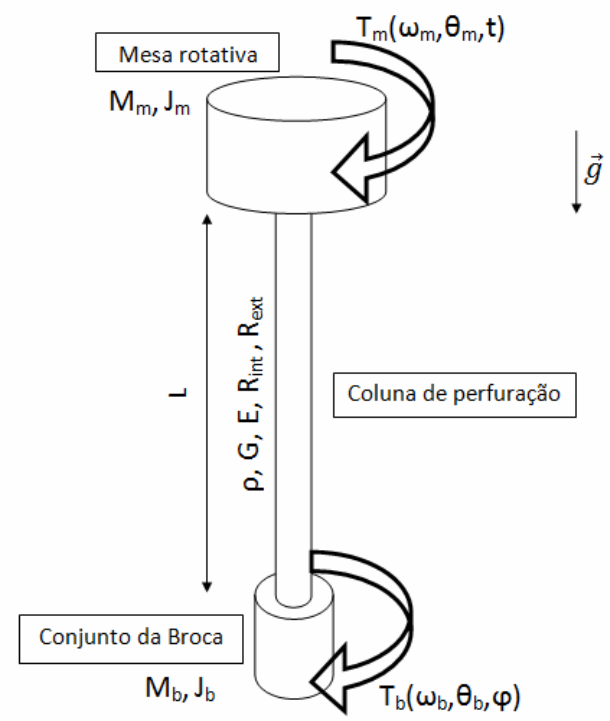

Figura 4 - Esquema representativo do modelo do sistema de perfuração, envolvendo a mesa rotativa, o conjunto da broca e a coluna de perfuração. 
A coluna é discretizada pelo método dos elementos finitos com funções de forma de Lagrange lineares para os deslocamentos angulares das seções da coluna $\theta(x, t)$.

Na tabela 2, encontram-se os itens do modelo composto.

Tabela 2 - Quadro-resumo para a modelo considerado para o sistema de perfuração, divido em coluna de perfuração, torque na mesa e torque na broca.

\begin{tabular}{cl}
\hline Item do modelo & \multicolumn{1}{c}{ Modelagem } \\
\hline $\begin{array}{c}\text { Coluna de } \\
\text { perfuração }\end{array}$ & $\begin{array}{l}\text { Modelada em elementos finitos, com } \\
\text { função de forma linear. }\end{array}$ \\
& $\begin{array}{l}\text { Controlador PI atuante sobre a } \\
\text { velocidade angular na mesa, } \\
\text { propiciando ganhos para a velocidade } \\
\text { na mesa (Proporcional) e para os } \\
\text { deslocamentos (Integrativo). }\end{array}$ \\
Torque na mesa na broca & $\begin{array}{l}\text { Modelo não regularizado para o torque } \\
\text { segundo atrito de Coulomb, pela } \\
\text { proposta de Navarro-López e Suárez } \\
\text { (2004). }\end{array}$ \\
\hline
\end{tabular}

\subsection{Influência dos parâmetros $K p$ e $K i$ e WOB no Controle PI}

Observou-se, que o parâmetro integral da velocidade $(K i)$, proporcional ao deslocamento, afeta a rigidez equivalente do sistema. Assim, o seu aumento induz um aumento na frequência de oscilação das velocidades da mesa e da broca. $\mathrm{O}$ parâmetro proporcional à velocidade afeta o amortecimento equivalente do sistema, um aumento no $K p$ então propicia um decaimento mais acentuado das amplitudes de vibração do sistema, como observado por Manzato (2011).

Pode-se verificar a influência do $W O B$ na amplitude das oscilações de velocidade. Ao aumentar-se o $W O B$, maiores se tornam as amplitudes de velocidades na mesa e na broca, portanto, a mais instabilidades a coluna de perfuração pode estar sujeita.

\subsection{Controlador PI com peso na broca} variável

Este controlador utiliza-se de uma variação linear do peso na broca, foi proposto para buscar reduzir oscilações, ou fazer desprender novamente o conjunto da broca, alterando-se o valor da força de atrito, que depende deste peso.

A variação linear do peso na broca segue a equação 6, em que: $W O B$ é o peso na broca variável, em newtons; $W O B_{0}$ é o peso na broca fixo no tempo; $K_{W}$, o parâmetro de controle de variação do peso na broca, em N.s; $\omega_{m}$, velocidade angular da mesa, em rad/s; $\omega_{\text {ref }}$, velocidade angular de referência para a coluna de perfuração, $\mathrm{em} \mathrm{rad} / \mathrm{s}$.

$$
W O B=W O B_{0}+K_{W} \cdot\left(\omega_{m}-\omega_{r e f}\right)
$$

\subsubsection{2a $2^{\mathrm{a}}$. Técnica de Controle}

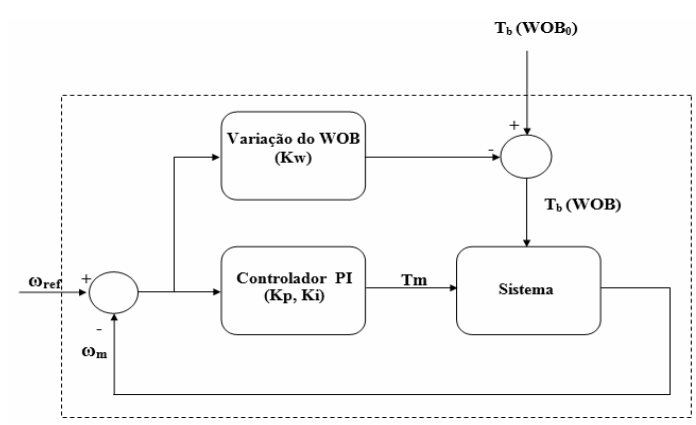

Figura 5 - Modelo esquemático de um controlador PI com peso na broca variável, adotado para o sistema de perfuração.

$\mathrm{Na}$ figura 5, encontra-se representado um modelo esquemático para esta implementação, que, combinada ao controlador PI, constitui a segunda técnica de controle considerada. As entradas deste controlador são: o peso na broca inicial e a velocidade de referência; a saída: a velocidade angular da mesa, sendo o retângulo em tracejado o sistema controlado.

\section{Métodos}

Foram adotados previamente critérios para avaliação de desempenho e de viabilidade e segurança de operação.

Os critérios de avaliação adotados foram: $J_{l}$, Desvio médio de velocidade angular na broca; $J_{2}$, Taxa de penetração média relativa.

Os critérios de segurança: $J_{3}$, Tensão de cisalhamento máximo na coluna; $J_{4}$, Desvio máximo de velocidade angular na broca; $J_{5}$, Torque máximo na broca; $J_{6}$, Potência máxima exigida do motor.

Para avaliação dos critérios adotados, foram utilizadas malhas de valores para $\mathrm{Kp}$ e $\mathrm{Ki}$, no caso, os critérios $J_{2}, J_{3}, J_{4}, J_{5}, J_{6}$, foram avaliados para a faixa de valores de $K p$ e $K i$ com $K p$ de 0 a 1000 N.m.s e variação de 100 N.m.s, e $K i$ de 0 a 1000 N.m, com variação de 100 N.m.

Verificou-se que, em ajuste dos parâmetros Kp e Ki, relatados a seguir no item 3.2, nas situações dos melhores valores de $J_{I}$ (os menores desvios), os demais critérios considerados não exerciam influência significativa. Isso foi constatado ao se avaliar cada outro critério em conjunto com $J_{l}$, na obtenção das curvas por análise paramétrica, o que produziu resultados semelhantes.

Foram considerados os resultados apenas para o critério de análise do desvio médio de velocidade angular na broca, por apresentar-se o mais significativo, e este é descrito no item 3.1. 


\subsection{Desvio médio de velocidade angular da broca $\left(J_{1}\right)$}

Este índice quantifica um desvio médio da velocidade angular da broca $\left(\omega_{b}\right)$ em relação à velocidade de referência ( $\left.\omega_{r \ell f}\right)$, constituindo-se da soma dos desvios pontuais em cada instante de tempo $t$, descrito matematicamente na equação 47 :

$$
J_{1}=\int_{t_{i}}^{t_{f}} \frac{\left|\omega_{b}-\omega_{r e f}\right|}{\omega_{r e f}} d t
$$

O desvio médio de velocidade angular propiciou resultados significativos e favoráveis para condicionar a escolha dos parâmetros $K p$ e $K i$, pois, para tal critério, houve a formação de regiões bem delineadas, em que, no seu interior, a velocidade angular apresentasse determinado desvio.

Do item 3.2 em diante os resultados considerados o são para o desvio médio da velocidade angular, $J_{l}$. Havendo citações de "desvio", sem especificação, estas referem-se ao desvio $J_{l}$.

\subsection{Ajuste dos parâmetros $K p$ e $K i$}

Foram criadas malhas paramétricas de pares destes parâmetros para cada peso na broca, e percebeu-se, nestas malhas, que alguns pares minimizavam o desvio, proporcionando maiores ou menores valores para o desvio, conforme o par de valores de $K p$ e $K i$. Em alguns casos, o tempo de acomodação ao valor da velocidade angular de referência era menor do que 100 segundos.

Em outros casos, verificou-se se que poderia convergir para a referência em um tempo maior do que 100s, e, ainda, outros pares de $K p$ e $K i$ poderiam provocar grandes oscilações às velocidades da mesa e da broca.

Verificou-se a formação de figuras semelhantes a elipses, que delimitavam uma região em que, no seu interior, os pares de $K p$ e $K i$ propiciavam determinado desvio médio da velocidade, $J_{l}$.

\section{Resultados e Discussões}

\subsection{Curvas de nível para PI com WOB} constante

Foram obtidas curvas de nível para o desvio $J_{l}$, considerando-se intervalos iguais de $K p$ e $K i$, foram obtidas as curvas de nível para desvios $J_{l}$ iguais a $10,20,30,40,50$, para os pesos na broca considerados $(80,100,120,140$ e $160 \mathrm{kN})$. Para todos os casos foi considerado o mesmo intervalo de $K p$ e $K i$, com variação de 50 N.m.s para $K p$ e 50 N.m para $K i$. Os resultados para esta comparação são apresentados na figura 45 .

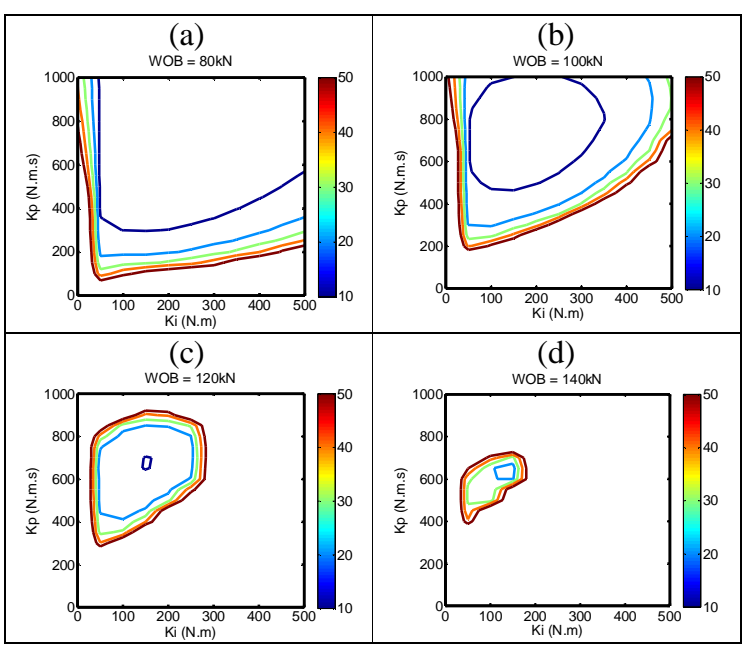

Figura 6 - Quadro comparativo das curvas de nível obtidas por Controle PI para diferentes valores de peso na broca.

A figura 6 apresenta os gráficos para os pesos na broca iguais a $80,100,120$ e $140 \mathrm{kN}$, sendo que, para $W O B=160 \mathrm{kN}$, não foram encontradas curvas para a malha considerada, resultando em um gráfico vazio, e por isso, não apresentado.

Por este quadro comparativo, pode-se ver a diminuição das regiões delimitadas pelas curvas referentes aos desvios $J_{l}$ com o aumento do $W O B$. Visualiza-se a diminuição da curva de $J_{l}=10$, de (a) para (b) e de (b) para (c), sendo que, em (d), a curva para este valor de desvio já não existe.

Para o Peso na broca $(\mathrm{kN})$ igual a $80 \mathrm{kN}$, as regiões que propiciam menor desvio apresentam-se delimitadas pelas curvas na cor azul, e pontos interiores a estas regiões (de desvios menores do que 10 e 20) propiciam minimização ou eliminação do problema de oscilações acentuadas e do stickslip. Tais curvas ficam interiores ao intervalo com $K p$ de 150 a 2200N.m.s de $K i$ de 100 a 900N.m.

Para o Peso na broca $(\mathrm{kN})$ igual a $100 \mathrm{kN}$, as curvas de desvio $J_{l}$ de 10 até 60 apresentam-se em faixas regulares, e houve uma redução de $84 \%$ em relação à área que delimita os mesmos desvios para $80 \mathrm{kN}$. Para as curvas de menor desvio, que propiciam as melhores condições de operação, com $J_{l}$ igual a 10 e 20 , houve uma redução de área de abrangência de $79 \%$.

Para o peso na broca igual a $160 \mathrm{kN}$, não foram encontradas faixas regulares do comportamento das curvas de desvio, nem curvas para os desvios menores do que 30, indicando o colapso destas regiões, em comparação aos resultados obtidos para menores pesos na broca. Mesmo após os refinamentos nas malhas, não foi encontrada uma região adequada de trabalho, apenas de valores pontuais de $K p$ e $K i$ que propiciam menor desvio. 
4.2 Resultados obtidos para PI com WOB variável e comparação entre as duas técnicas de Controle

No item 4.2.1, tratam-se da análise pontual para diferentes pares de valores de $K p$ e $K i$ (pontos), com melhor valor de $K w$ obtido para cada ponto, considerando-se o critério de desvio $J_{l}$. Obtém-se gráficos de alguns pontos e analisam-se a alteração no desvio $J_{l}$ para o peso na broca constante e peso na broca variável.

4.2.1 Análise pontual para estudo comparativo da influência da variação do peso na broca

Foram escolhidos quatro pares de valores para $K p$ e $K i$ com diferentes desvios, além do ponto ótimo para cada $W O B$. O critério para escolha destes pontos foi utilizarem-se os pontos de um retângulo imaginário para os pares de valores de $K p$ e $K i$, de menor tamanho possível, situando o ponto ótimo obtido para a malha paramétrica de $K p$ e $K i$ próximo ao centro deste retângulo, e cujos vértices, não necessariamente todos, apresentassem desvios $\mathrm{J} 1$ maiores do que 30 .

Para comparação dos resultados de todos os desvios pontos adotados, considerando-se as duas técnicas de Controle, PI com $W O B$ constante e PI com $W O B$ variável, os mesmos são apresentados na tabela 3, para os pontos (1), (2), (3) e (4) bem como dos pontos ótimos (P.O.), sendo estes diferentes para cada peso na broca.

Tabela 3 - Relação comparativa dos desvios $J_{l}$ obtidos para as duas técnicas de controle propostas para os pares de valores dos parâmetros $K p$ e $K i$.

\begin{tabular}{|c|c|c|c|c|c|}
\hline \multirow{4}{*}{$\begin{array}{c}\text { WOB } \\
(\mathrm{kN})\end{array}$} & \multicolumn{4}{|c|}{ Desvio $J_{l}$} & \multirow{4}{*}{$\begin{array}{c}\text { Redução } \\
\text { do } \\
\text { desvio } \\
\text { J1 em }\end{array}$} \\
\hline & & & PI com & $K_{w}$ & \\
\hline & & PI & WOB & $\mathrm{KW}$ & \\
\hline & & & variável & & \\
\hline \multirow{5}{*}{80} & (1) & 42.83 & 17.7 & 13.7 & $59 \%$ \\
\hline & (2) & 39.24 & 22.59 & 13.5 & $42 \%$ \\
\hline & (3) & 18.02 & 13.07 & 1 & $27 \%$ \\
\hline & (4) & 50.15 & 8.62 & 3.1 & $83 \%$ \\
\hline & P.O. & 3.78 & 3.68 & 0.5 & $3 \%$ \\
\hline \multirow{5}{*}{100} & (1) & 15.26 & 10.07 & 1.8 & $34 \%$ \\
\hline & (2) & 62.21 & 7.08 & 3.7 & $89 \%$ \\
\hline & (3) & 62.95 & 58.17 & 3.5 & $8 \%$ \\
\hline & (4) & 44.79 & 32.00 & 2.7 & $29 \%$ \\
\hline & P.O. & 5.84 & 5.74 & 0.5 & $2 \%$ \\
\hline \multirow{5}{*}{120} & (1) & 14.21 & 10.92 & 1.9 & $23 \%$ \\
\hline & (2) & 63.49 & 7.15 & 3.9 & $89 \%$ \\
\hline & (3) & 65.38 & 63.99 & 2.7 & $2 \%$ \\
\hline & (4) & 42.72 & 26.08 & 1.6 & $39 \%$ \\
\hline & P.O. & 9.73 & 8.21 & 1.4 & $16 \%$ \\
\hline \multirow{5}{*}{140} & (1) & 21.87 & 18.31 & 0.5 & $16 \%$ \\
\hline & (2) & 64.26 & 15.06 & 1.4 & $77 \%$ \\
\hline & (3) & 68.78 & 68.09 & 2.5 & $1 \%$ \\
\hline & (4) & 72.99 & 68.37 & 2.7 & $6 \%$ \\
\hline & P.O. & 16.12 & 15.78 & 0.2 & $2 \%$ \\
\hline \multirow{5}{*}{160} & (1) & 69.88 & 57.69 & 0.4 & $17 \%$ \\
\hline & (2) & 83.48 & 48.13 & 0.6 & $42 \%$ \\
\hline & (3) & 59.79 & 59.79 & 0 & $0 \%$ \\
\hline & (4) & 76.55 & 59.65 & 0.4 & $22 \%$ \\
\hline & P.O. & 35.39 & 35.39 & 0 & $0 \%$ \\
\hline
\end{tabular}

Analisando-se tais resultados apresentados na tabela 3, observou-se que a implementação do peso na broca variável provocou redução do desvio médio de velocidade angular $\left(J_{1}\right)$ em $92 \%$ dos casos tratados.

Não foi encontrado valor de $K w$ maior que 0.01 que propiciasse alteração no desvio nos restantes 8\%. Dos 16 casos, entre os 25 pontos considerados, pela implementação do peso na broca variável foi possível a eliminação das oscilações/stick-slip em sete pontos, propiciando um sucesso efetivo de $43 \%$, em relação a esta implementação e o modo como foram obtidos tais pontos.

$\mathrm{Na}$ figura 7, apresentam-se as curvas dos desvios dos pontos ótimos obtidos com e sem a variação do peso na broca, para efeitos de comparação. Observam-se que, com o aumento do $W O B$, os desvios para os pontos ótimos aumentam, e tendencialmente, para determinado valor alto de peso na broca, já não será possível obter-se pontos de desvios $J_{l}$ relativamente baixos, em que as velocidades da mesa e da broca aproximem-se da velocidade de referência, diminuindo a amplitude das oscilações.

Verifica-se, para as curvas em vermelho (PI) e azul (PI com variação do $W O B$ ), na figura 51, uma similaridade entre as mesmas, tendo os desvios para o PI com variação do $W O B$ um pouco menores do que os obtidos com PI para $W O B$ constante.

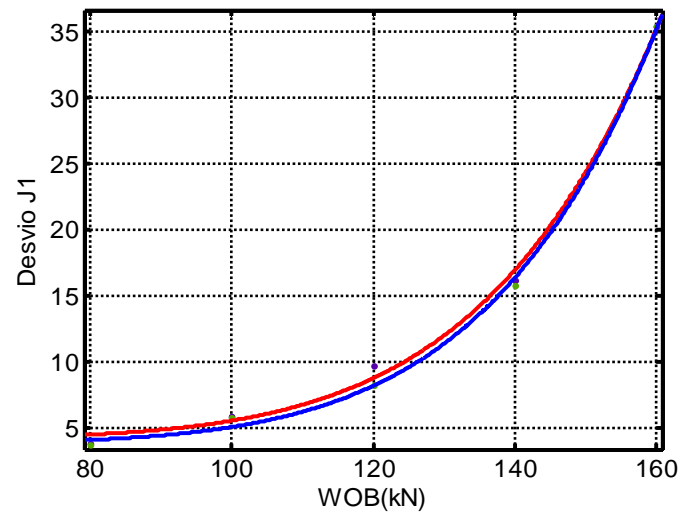

Figura 7 - Desvio médio da velocidade angular do ponto ótimo $\left(J_{l}\right)$ em relação ao $W O B(\mathrm{kN})$, em vermelho para PI, em azul para PI com $W O B$ variável.

Consequentemente, a variação do peso na broca, a partir de determinado valor de peso na broca inicial, por mais promova a diminuição do desvio pontual, não provoca o surgimento de pontos ótimos melhores do que os obtidos por controle PI, considerando-se o peso na broca constante.

Deve-se salientar que o ponto ótimo é o par de valores para $K p$ e $K i$ que propiciam o menor desvio $J_{l}$, mas à medida que o peso na broca é aumentado, 
este desvio também aumenta, e pode-se ter um ponto ótimo que não apresente efetiva aproximação à velocidade de referência, apresentando, ao contrário, elevadas oscilações e alta frequência de stick-slip.

Baseado nestas observações feitas sobre o comportamento das curvas de desvio na figura 7 , percebe-se que, adequando-se os parâmetros de $K p$ e $K i$ para os valores de menor desvio, a implementação da variação do peso na broca pouca influência exerce na diminuição do desvio médio da velocidade angular, $J_{l}$.

\subsubsection{Análise em curvas de nível para estudo comparativo da influência da variação do peso na broca}

Para $W O B=80 \mathrm{kN}$, com $K w=1$, foram obtidas falhas na obtenção das curvas de nível para o desvio $J_{l}$ relacionadas a este valor de $K w$, mostrando que $K w$ precisava ser menor do que o planejado inicialmente.

Adotou-se $K w=0.5$, e obtiveram-se as curvas de nível da malha paramétrica de $K p$ e $K i$ para este peso na broca, que está apresentada na figura 8 , em (b). Em (a), está reproduzida novamente, para efeitos de comparação, as curvas de nível obtidas para os mesmo peso na broca, constante $(K w=0)$.

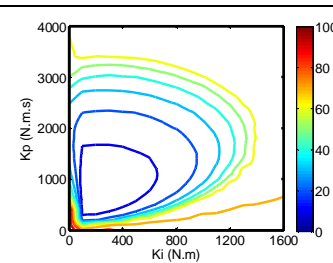

(a)

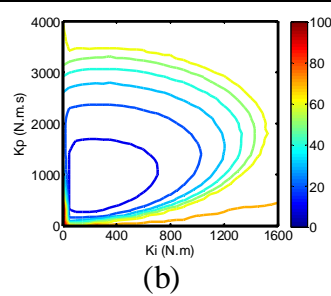

(b)
Figura 8 - Curvas de nível para $K p(0$ a 4000 N.m.s, com passo 100 N.m.s $)$ e $K i(0$ a 1600 N.m, com passo $50 \mathrm{~N} . \mathrm{m})$, com Controle PI e (a) $W O B=80 \mathrm{kN}$. (b) $W O B$ variável com $W O B_{0}=$ $80 \mathrm{kN}$ e $K w=0.5$.

Visualiza-se, na figura 8 , para $80 \mathrm{kN}$, que a variação do peso na broca, em (b), amplia todas as curvas de nível para o desvio $J_{1}$, aumentando a área de abrangência para todas as curvas, provocando a diminuição de forma geral do desvio neste limite dos parâmetros $K p$ e $K i$, com $K p$ de 0 a 4000N.m.s e $K i$ de 0 a 1600 N.m.

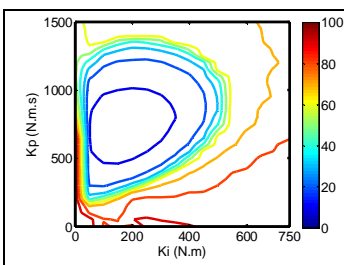

(a)

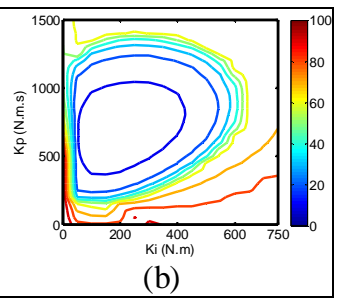

(b)
Figura 9 - Curvas de nível para $K p(0$ a 1500 N.m.s, com passo $50 \mathrm{~N} . \mathrm{m} . \mathrm{s})$ e $K i(0$ a $750 \mathrm{~N} . \mathrm{m}$, com passo $50 \mathrm{~N} . \mathrm{m})$ com Controle PI e (a) $W O B=100 \mathrm{kN}$. (b) $W O B$ variável, com $W O B_{0}=100 \mathrm{kN}$ e $K w=1.2$.
A figura 9 apresenta as curvas de nível para $100 \mathrm{kN}$, mantendo-se este peso na broca, em (a), e variando-o linearmente, a partir deste valor, em (b), com o fator de variação linear $K w=1.2$. Mudanças significativas foram encontradas na configuração das curvas.

Em (b), a curva de menor desvio $J_{1}=10$, amplia-se consideravelmente, alongando-se para a direita, no sentido crescente dos valores de $K i$, e também para cima e para baixo, resultando em uma maior faixa de valores de $K p$ e $K i$ para pontos interiores à curva. Isso equivale a dizer a implementação do peso na broca variável com este valor de $K w$, provocou a redução dos desvios para certo número de pontos, tornando pontos antes exteriores em interiores à curva de $J_{I}=10$.

Para outras curvas de desvios $J_{l}=10$ até 60 (do azul ao amarelo), ainda na figura 9, é possível visualizar uma ampliação das regiões delimitadas pelas mesmas; para $J_{l}=60$ (na cor amarela), o limite para $K i$ se estende, de 500N.m em (a) para 600 N.m em (b), bem como as curvas para 70 e 80 também são ampliadas.

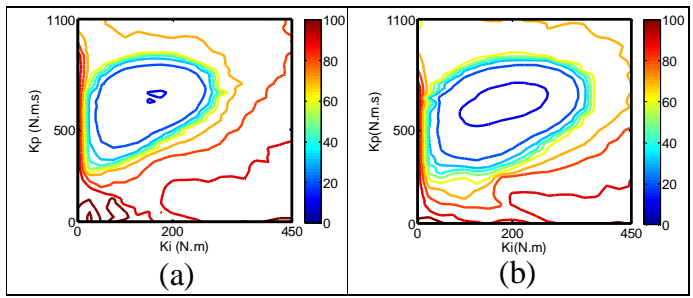

Figura 10 - Curvas de nível para $K p(0$ a 1100 N.m.s, com passo 25 N.m.s) e $K i(0$ a 450 N.m, com passo 25 N.m) com Controle PI e (a) $W O B=120 \mathrm{kN}$. (b) $W O B$ variável com $W O B_{0}=120 \mathrm{kN} \mathrm{e}$ $K w=1.4$.

Para o peso na broca de $120 \mathrm{kN}$, foram expressas, na figura 10, as curvas de nível para o desvio $J_{l}$ obtidas para o peso na broca constante e para o peso na broca variável, a partir deste valor, em (b), com Controle PI. De (a) para (b) houve uma ampliação da região delimitada pela curva de desvio $J_{l}=10$, extremamente reduzida em (a), e apresentando contornos bem definidos em (b), nos limites para $K p$ e $K i$ com $K p$ de 500 a 750N.m.s e $K i$ de 100 a 300N.m.

Houve, também, de (a) para (b) na figura 10, um relativo aumento das áreas de abrangência de todas as curvas. A curva em amarelo, que representa $J_{1}=60$, tornou-se mais regular, suave, em (b), estendendo-se, bem como todo o conjunto de curvas para desvios menores em seu interior, de $K i=300$ N.m para $K i=400$ N.m, e as curvas de nível em laranja $\left(J_{1}=70\right)$, alongaram-se de $K i=$ 350 N.m para $K i=450$ N.m, aproximadamente. 


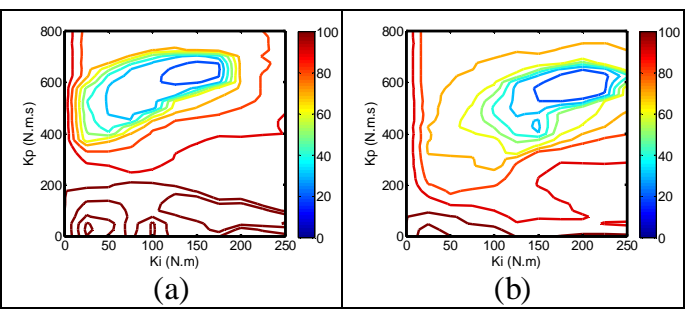

Figura 11 - Curvas de nível $K p$ (0 a 800N.m.s, com passo $25 \mathrm{~N} . \mathrm{m} . \mathrm{s})$ e $K i$ ( 0 a $250 \mathrm{~N} . \mathrm{m}$, com passo $25 \mathrm{~N} . \mathrm{m})$ com Controle PI e (a) $W O B=140 \mathrm{kN}$. (b) $W O B$ variável com $W O B_{0}=140 \mathrm{kN} \mathrm{e}$ $K w=1.7$.

Para avaliar por comparação de efeitos a influência da variação do peso na broca para $W O B$ $=140 \mathrm{kN}$, apresentam-se, na figura 11 , as curvas de nível para o desvio médio da velocidade angular $\left(J_{1}\right)$, obtidas com Controle PI e peso na broca constante, em (a) e peso na broca variável, a partir de $W O B_{0}=140 \mathrm{kN}$ e $K w=1.7$, em (b).

A variação linear do peso na broca proporcionou um aumento relativo da área formada pela curva $J_{l}=20$, de (a) para (b), na figura 11 . Proporcionou, no entanto, uma diminuição da área para desvio $J_{1}=30$, deslocando-a, ainda, para a direita, para valores crescentes de $\mathrm{Ki}$, aumentandose a frequência de oscilações das velocidades. Já a curva de desvio $J_{1}=70$ (em laranja) dilatou-se consideravelmente, de (a) para (b).

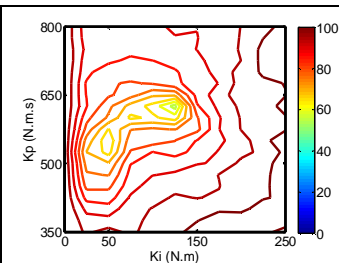

(a)

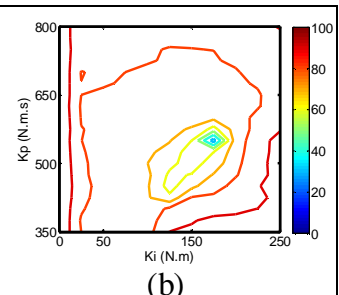

(b)
Figura 12 - Curvas de nível $K p(350$ a 800N.m.s, com passo 25 N.m.s) e $K i(0$ a 250 N.m, com passo 25 N.m) com Controle PI e (a) $W O B=160 \mathrm{kN}$. (b) $W O B$ variável com $W O B_{0}=160 \mathrm{kN} \mathrm{e}$ $K w=2$.

Para $\mathrm{WOB}=160 \mathrm{kN}$, nota-se, na figura 12 , que a variação do peso na broca conseguiu diminuir os desvios de velocidade para a malha considerada, e fez aparecer uma pequenina região em azul mais claro, representando desvios menores do que 30.

$\mathrm{Na}$ primeira figura, em (a), a malha considerada não é suficiente para fazer aparecer regiões de desvio menores, notificando que o ponto ótimo para esta malha, de desvio $J_{1}=35$, é para o par $K p=512$ N.m.s e $K i=63$ N.m, de cuja região encontra-se circunscrita a outra de desvio 70, identificável apenas após refinamento; o que mostra tratar-se de um caso pontual de relativamente baixo desvio, considerando-se a dificuldade de se encontrar regiões de desvios baixos para este valor de peso na broca.

Verifica-se, em (b), com o surgimento da região em azul claro representado desvios $\mathrm{J}$ 1 menores do que 30, situada para valores de $K i$ próximos a $170 \mathrm{~N} . \mathrm{m}$, que o ponto ótimo foi deslocado para a direita, em relação ao obtido com peso na broca constante.

Por outro lado, a variação do peso na broca diminuiu os desvios em caráter geral para a malha considerada; a região para $J_{l}=70$ foi diminuída e deslocada para a direita, enquanto que a curva em amarelo apresentou um aumento considerável. As curvas apresentadas em (b) apresentam-se mais bem delineadas e regulares.

\subsection{Resumo dos resultados obtidos}

Utilizando-se a técnica de controle Proporcional Integrativo com WOB constante, foram obtidas regiões bem delineadas de determinado desvio $J_{l}$, em formas mais regulares quanto menor o peso na broca. As malhas paramétricas consideradas foram menores conforme o peso na broca, e de cada malha, foi possível, conforme a discretização, encontrar-se um ponto ótimo, ponto de menor desvio para a malha considerada.

Aumentando-se o peso na broca, as regiões limitadas pelas curvas de desvio se fecham consideravelmente, e a região de trabalho adequada no sentido de baixas oscilações nas velocidades e ausência ou minimização do fenômeno de stickslip, torna-se cada vez mais restrita, extinguindo-se a partir de determinado valor de peso na broca; no caso, para $160 \mathrm{kN}$.

Enquanto que, para a $2^{\mathrm{a}}$. Técnica de Controle, a implementação da variação do peso na broca apresentou-se como auxiliar do Controle PI, diminuindo pontualmente os desvios obtidos com $W O B$ constante, obtendo-se para tanto, o melhor valor do parâmetro $K w$ que minimizasse $J_{l}$.

Foi observado um aumento das regiões de desvio de forma geral, nas malhas consideradas, aumentando as áreas de menor de desvio, principalmente quanto menor o peso na broca.

Pesos na broca maiores provocaram, por um lado, o aumento de algumas regiões e a diminuição de outras, ainda também as deslocando para a direita, no sentido de aumento dos valores do parâmetro $K i$, o que consequentemente, aumenta a frequência das oscilações das velocidades.

A implementação do peso na broca variável não alterou significativamente os desvios obtidos dos pontos ótimos obtidos com WOB constante.

\section{Discussões e Conclusões}

A construção de malhas paramétricas dos parâmetros integral e proporcional, para os desvios médios de velocidade, para os diferentes pesos na broca considerados apresentou-se útil aos objetivos deste trabalho, avaliar as duas técnicas de controle 
apresentadas bem como investigar a possibilidade de encontrar-se regiões bem comportadas de velocidade.

A dificuldade encontrada foi a conveniente adequação dos valores dos parâmetros proporcional, integral e de variação do peso na broca, com o definir a melhor maneira de realizarse uma conveniente análise paramétrica.

Em relação a trabalhos futuros, a avaliação de desempenho para outras técnicas de Controle, como, o Soft Torque, que envolve o conceito de ondas torcionais, poderia ser utilizada.

As vibrações axiais e laterais poderiam ser consideradas, o que aumentariam as dificuldades na implementação numérica, e um trabalho com uma análise paramétrica mais ampla também pode ser considerado.

\section{Agradecimentos}

Agradecemos à Universidade de São Paulo, bem como ao Programa de Pós-Graduação em Engenharia Mecânica, bem como ao professor orientador Prof. Dr. Marcelo Areias Trindade pela oportunidade a nós fornecida.

Agradecemos também à Universidade Federal de São Carlos, ao Centro de Ciências Exatas e Tecnologia, ao curso de Engenharia Mecânica desta instituição, à qual estamos vinculados profissionalmente, por ceder-nos o tempo necessário para realização deste artigo.

\section{Referências Bibliográficas}

Jansen, J.D. and van den Steen, L., 1995. "Active damping of self-excited torsional vibration in oil well drillstrings". Journal of Sound and Vibration, Vol. 179, No. 4, pp. 647-668. DOI: 10.1006/jsvi.1995.0042

Navarro-López, M.N. and Suarez, R., 2004. "Practical approach to modeling and controlling stick-slip oscilations in oilwell drillstring". Proceedings of the 2004 IEEE International Conference on Control Applications, Taipei, Taiwan, pp. 1454-1460.

Placido, J.C.R., Santos, H.M.R. and Galeano, Y.D., 2002. "Drillstring vibration and wellbore instability". Journal of Energy Resources Technology, Vol. 124, pp. 217-222. DOI: $10.1115 / 1.1501302$

Richard, T. and Detournay, E., 2000. "Stick-slip motion in a friction oscillator with normal and tangential mode coupling". Comptes Rendus de l'Académie des Sciences - Série IIb, Vol . 328, pp. 671-678. DOI: 10.1016/S1620-7742(00)01240-X

Ritto, T.G., Soize, C. and Sampaio, R., 2009. "Non-linear dynamics of a drill-string with uncertain model of the bit-rock interaction". International Journal of Non-Linear Mechanics, Vol. 44, pp. 865-876. DOI: 10.1016/j.ijnonlinmec.2009.06.003

Schlumberger Ltd., 2006. "Shock and vibration in the drilling environment". Tutorial video.
$8 \min 20 \mathrm{~s}$.

http://www.slb.com/services/drilling.aspx.

Trindade, M.A. and Sampaio, R., 2005. "Active control of coupled axial and torsional drillstring vibrations". Proceedings of 18th COBEM International Congress of Mechanical Engineering, Ouro Preto, MG, in CD-ROM.

Tucker, R.W. and Wang, C., 1999a. "On the effective control of torsional vibrations in drilling systems". Journal of Sound and Vibration, Vol. 224, No. 1, pp. 101-122. DOI: 10.1006/jsvi.1999.2172

Tucker, R.W. and Wang, C., 1999b. "An integrated model for drill-string dynamics". Journal of Sound and Vibration, Vol. 224, No. 1, pp. 123-165. DOI: 10.1006/jsvi.1999.2169

Tucker, R.W. and Wang, C., 2003. "Torsional vibration control and cosserat dynamics of a drill-rig assembly". Meccanica, Vol. 38, pp. 143-159. DOI: 10.1023/A:1022035821763

Álamo, F. J. C., 2004. "Vibrações Axiais e Torcionais em Colunas de Perfuração." Exame de Qualificação (Doutorado em Engenharia Mecânica) - Departamento de Engenharia Mecânica da Pontifícia Universidade Católica do Rio de Janeiro, Rio de Janeiro, 2004.

Christoforou, A. P.; Yigit, A. S., 2003. "Fully coupled vibrations of actively controlled drillstrings". Journal of Sound and Vibration, v. 267, p.1029-1045, $2003 . \quad$ DOI: 10.1016/S0022-460X(03)00359-6

Coletti, A. Y. Y., 2011. "Dinâmica e controle de vibrações torcionais em colunas de perfuração de poços de petróleo." São Carlos: Departamento de Engenharia Mecânica, 2011. 53 p. Relatório Técnico de Iniciação Científica.

Khulief,, Y. A.; Al-Naser, 2005. "H. Finite element dynamic analysis of drillstrings." Finite Elements in Analysis and Design, v. 41, p. 1270-1288, 2005. DOI: 10.1016/j.finel.2005.02.003

Manzatto, L. M., 2011. “Análise dinâmica colunas de perfuração de poços de petróleo usando controle de velocidade não-colocalizado." 2011. 112 p. Dissertação (Mestrado em Engenharia Mecânica) - Departamento de Engenharia Mecânica, USP, São Carlos, 2011.

Mihajlovic, N. et al., 2006. "Friction-induced limit cycling in flexible rotor systems: an experimental drill-string set-up." Nonlinear Dynamics, v. 46, n. 3, p. 273-291, 2006. DOI: 10.1007/s11071-006-9042-z

Monteiro, H. L. S. "Análise de desempenho de diferentes leis de controle de vibrações torcionais em colunas de perfuração de poços de petróleo." 2012. 125p. Dissertação (Mestrado em Engenharia Mecânica) Departamento de Engenharia Mecânica, USP, São Carlos, 2012. 